https://doi.org/10.32541/recie.2017.v1i1.pp34-52

\title{
ESTRATEGIAS DE ACOMPAÑAMIENTO PEDAGÓGICO PARA EL DESARROLLO PROFESIONAL DOCENTE
}

\section{Educational support strategies for teachers' professional development}

\section{- Bismar Galán}

Instituto Superior de Formación Docente

Salomé Ureña, República Dominicana

Correo-e: galanbismar@yahoo.es

\section{Resumen}

Entre los múltiples retos que se plantean a la educación como herramienta para el logro de una verdadera ciudadanía, uno de los más justificados es la profesionalización de los colectivos pedagógicos. En tal intención, el acompañamiento pedagógico es una herramienta que agrupa múltiples estrategias dirigidas al desarrollo de competencias docentes, a partir del trabajo coordinado y según las necesidades y fortalezas individuales. En el presente artículo, derivado de un estudio que buscaba analizar las estrategias de acompañamiento pedagógico empleadas en dos centros educativos de primaria de Santo Domingo, República Dominicana; se intenta invitar a la reflexión sobre del rol que juegan dichas estrategias en el desarrollo profesional docente. Para el estudio se empleó el enfoque cualitativo, ya que la intención era profundizar en casos específicos; la prioridad fue cualificar y describir el fenómeno objeto de estudio a partir de rasgos determinantes. En correspondencia, se optó por la entrevista no estructurada, el grupo focal y el análisis documental. Los resultados revelan pobre participación de los docentes en el proceso, transgresión de momentos del acompañamiento, falta de diversidad y carácter sistemático en las actividades y pobre cohesión en su desarrollo. Se concluyó que esta estrategia de trabajo no se aprovecha en los centros educativos estudiados, como vía para incidir en el desarrollo continuo de competencias básicas del docente en ejercicio.

Palabras clave: acompañamiento pedagógico; docente; desarrollo profesional.

\begin{abstract}
Among the multiple challenges that are posed to education as a tool for the achievement of true citizenship, one of the most justified is the professionalization of pedagogical groups. In this matter, the pedagogical follow up is a tool that merges multiple strategies aimed to the development teaching skills based on coordinated work plan and in accordance to individual needs and strengths. In the present article, as a result of a study that sought to analyze the pedagogical accompaniment strategies used in two primary schools of Santo Domingo, it is intended to encourage people to reflect on the role that strategy plays in the professional development of teachers. For the study, the qualitative or non-traditional approach was used, since the intention was to delve into specific cases; the priority was to qualify and describe the phenomenon under study based on determining features. In correspondence, we chose the non-unstructured interview, the focus group and the documentary analysis. The results reveal poor participation of teachers in the process, violation of moments of accompaniment, lack of diversity and system nature in the activities and poor cohesion in their development. It was concluded that this work strategy under-exploited in the studied educational centers, as a way to influence the continuous development of basic skills of the practicing teacher. Keywords: educational support; professional; development teachers.
\end{abstract}

ISSN (impreso): 2636-2139

ISSN (en línea): 2636-2147

Recibido: 25/04/2017

Sitio web: https://revistas.isfodosu.edu.do/recie

Aprobado: 20/06/2017

COMO CITAR:

Galán, B. (2017). Estrategias de acompañamiento pedagógico para el desarrollo profesional docente. Revista Caribeña de Investigación Educativa (RECIE), 1(1), 18-33. https://doi.org/10.32541/recie.2017.v1i1.pp34-52 


\section{Introducción}

El presente documento se basa en una investigación realizada en dos centros educativos de Santo Domingo (uno público y otro pivado), con la intención de estudiar la estrategia de acompańamiento pedagógico como vía para el desarrollo profesional de los maestros en ejercicio. Basado en los resultados de la referida investigación y en la experiencia acumulada por más de 25 ańos de trabajo en el tema, el presente artículo intenta provocar la reflexión sobre el rol que juega el acompañamiento pedagógico en la formación continua del docente. Se hace énfasis en el papel de esta estrategia de trabajo en los centros educativos, como vía para incidir en el desarrollo continuo de competencias básicas de los maestros y para el ejercicio eficiente de su labor pedagógica a favor del aprendizaje de sus estudiantes.

Hablar de acompañamiento pedagógico es hacer referencia a una de las variantes de la formación didáctica que corresponde a la labor científico-técnica en la educación primaria dominicana. La coordinación y ejecución de esta estrategia de formación docente están bajo la responsabilidad de los miembros de los equipos de gestión (coordinador y director) de los centros educativos.

La organización del acompañamiento pedagógico se ha de subordinar a la determinación (concepción consciente y objetiva) y planificación del sistema de acciones de desarrollo profesional a ejecutar en el centro educativo. Son vitales dichas determinación y planificación, previo al desarrollo; pero, para comprender el papel que desempeña dicho acompañamiento en la formación permanente del docente, es preciso tomar en cuenta cómo es definido y asumido dicho concepto, y sobre todo, cómo es vista su organización por los diversos actores a nivel de centro educativo.

Algo de vital importancia en la intención de lograr eficiencia en el trabajo integral del centro educativo es la participación de todas las personas involucradas en la organización de las acciones de enriquecimiento personal e institucional. Las actividades dirigidas al logro de la calidad educativa en el Nivel Primario en República Dominicana, de forma directa o indirecta, rozan lo concerniente al desarrollo didáctico de los maestros, aunque no siempre estos sean actores en cada una de las etapas de las acciones. Aun cuando desde las instancias superiores se exige la participación de todos, no siempre es así; por ejemplo, en los Planes de Mejora de los centros educativos que exige el Ministerio de Educación, donde el acompañamiento pedagógico debería ocupar un lugar primordial, se hace referencia a la necesidad de involucrar a todos: "Organizar reuniones para elaborar el Plan de Mejora con la participación de directivos, docentes, familias, estudiantes y la comunidad” (SEE, 2007, p. 8). Sin embargo, en la práctica, muchos docentes son simples receptores de lo previsto desde los equipos de gestión de los centros educativos y/o instancias superiores.

De ahí que se considere de mucho valor conocer cuál es la percepción de los diferentes actores de centros educativos primarios, acerca de las acciones de desarrollo profesional del docente, y como parte de ellas, la estrategia de acompańamiento pedagógico. De ese modo se accederían elementos a considerar en la toma de decisiones respecto a la concepción, planificación, organización, desarrollo y evaluación de las actividades formativas dirigidas al docente primario en ejercicio.

\section{Revisión de la literatura}

Como parte del trabajo de investigación realizado para describir la percepción de los actores de centros educativos primarios sobre el acompańamiento pedagógico como herramienta para el 
desarrollo profesional docente, se consultó una serie de trabajos de especialistas en el tema y en áreas interconectadas con este. Entre otras, acerca de la superación del maestro, fueron tomadas en cuenta, las ideas de Marcelo y Vaillant (2009), Mejía (2005), Almánzar (2008), Reimers y Tiburcio (1994); sobre las vías de superación del maestro se consideraron las aportaciones de Valle y García (2007), Matos Moquete (2013); mientras que sobre el acompañamiento pedagógico se apeló a los criterios de Aguerrondo y Xifra (2002), Jacobo (2005), MINERD (2010), Mañú y Goyarrola (2011), y Wanda Rodríguez (2013), entre los más destacados.

\subsection{Acompañamiento pedagógico: concepciones}

Para comprender lo concerniente al acompañamiento pedagógico como herramienta para la formación docente es pertinente partir de las múltiples concepciones que se esgrimen acerca de él. Para Osto (2006), el acompañamiento pedagógico es una mediación formativa basada en una relación de ayuda en la que se propicia que la persona crezca y madure en consistencia, responsabilidad y libertad. Por su parte, Planela (2009) plantea que el acompańamiento es un proceso que sirve para compartir con otro, o con otros el propósito de llegar a una meta o de conseguirla conjuntamente. Mientras, Soto (2011) asume que se trata de un proceso complejo y sistemático de apoyo, asesoramiento, formación y seguimiento que vincula diferentes equipos conformados en comunidades de aprendizaje comprometidas en mejorar los procesos de los centros educativos.

Jacobo (2005) ven al acompañamiento pedagógico como "la relación entre dos o más personas en procesos de enseñanza recíproca y de ayuda mutua, que procesan información o que adquieren y desarrollan otro tipo de competencias tanto en el nivel individual como en el colectivo"” (p. 9). En tanto que, investigadores del contexto dominicano (Martínez \& González, 2010) lo ven como mediación de formación en centro y para la vida, desde donde se recrea la dinámica relacional de la acción educativa y se aporta sentido vinculante a los nuevos conocimientos y competencias docentes.

Si se apunta a la necesidad de interacción del sujeto que aprende en colaboración con otros, el acompañamiento, a decir de Maturana (1997), tiene la virtud de la adecuación para dar atención y responder en diferentes direcciones y grados de asistencia según se requiera. Este actúa por demanda y se inserta en una red de relaciones colaborativas caracterizadas por su horizontalidad, retroacción y recursividad y, sobre todo, determinadas por un componente de calidad: la humanización de las relaciones sociales.

Jacobo y et al. (2005 p. 9) señalan que las personas que participan en las comunidades son acompañantes y acompañados a la vez, y su interacción auténtica requiere de confianza, respeto, tolerancia, igualdad, justicia, libertad, responsabilidad, autonomía y cooperación, creando un ambiente de aprendizaje y de intervención pedagógica pertinentes al entorno de la comunidad.

A partir de estas definiciones y posiciones teóricas se puede asumir el acompańamiento pedagógico como un proceso dinámico e interactivo de colaboración para el aprendizaje, según las necesidades individuales y las potencialidades del contexto. En un ambiente de respeto, cada individuo aporta desde sus saberes y experiencias, en beneficio de los demás miembros de su comunidad de aprendizaje.

En el acompańamiento cobra valor superior para el participante, la palabra soportada en criterios sólidos y trascendentes, que conduzca al accionar consciente y práctico. Jacobo y colaboradores, 
(2005) describen al docente sensible a la palabra informada y a la acción reflexionada, que puede desarrollar su trabajo de forma sistemática, tiene capacidad de pensar su trabajo, sobre y durante la acción, e innovar.

La actitud que asume el individuo, como acompañado o como acompañante, define en gran medida la efectividad del trabajo de formación del docente en su propia actividad profesional. Como afirmara Vygotsky (1978), la conducta del hombre, en primer lugar, está condicionada por el hecho de que el hombre interviene, activamente, en sus relaciones y, a través del medio. Él mismo modifica su conducta, sometiéndola a su poder. Dicha conducta definirá el grado en que el docente asume el rol que le compete en el acompañamiento pedagógico, lo que definirá si se cumple o no el objetivo previsto.

En su esencia, el acompañamiento pedagógico es un proceso de mediaciones que interconectan a los participantes. Como precisa Labarrere Sarduy (2008), cuando se habla de mediación pedagógica, se tiene en cuenta principalmente cierto género de acciones y actividades ejecutadas para obtener mejoras en las personas; estas acciones que pueden ser más o menos formales, están asociadas de alguna manera y en algún momento con la intencionalidad y la conciencia de los sujetos. De modo que quienes participan en el acompañamiento, en un proceso de aprendizaje compartido, van ganando conciencia de sus aportaciones y de sus asunciones en el proceso de formación profesional, individual y colectiva.

Dentro del acompańamiento cobra valor superior el diálogo reflexivo en la práctica; es decir, el diálogo crítico, apoyado en la experiencia y el desarrollo personal íntegro. En este, son de gran valor la escucha atenta, la coherencia y precisión en la expresión, el tono de voz con las inflexiones que requiere el discurso, la capacidad de reflexión, el respeto al otro, así como la tolerancia y empatía.

Por su parte, la indagación dialógica como elemento distintivo del acompañamiento pedagógico, presupone un conjunto de preguntas interconectadas que permiten profundizar en el debate a partir de las respuestas que van surgiendo. Mediante la indagación (Rodríguez, 2015) se busca la colaboración, la construcción del conocimiento en una comunidad profesional de aprendizaje colectivo, dentro de un ambiente de respeto mutuo. En ella se toma en cuenta tanto la experiencia personal y el contexto, junto con la afectividad que conlleva.

Como variantes del acompańamiento se pueden emplear el modelaje pedagógico y la tutoría. Este modelaje consiste en que un docente prepara y desarrolla una clase que es observada, analizada y debatida por el colectivo. Dicha actividad debe constituir un verdadero modelo a seguir por el resto de los docentes y, por tanto, debe ser rigurosamente preparada. Por su parte, y de acuerdo con Maya-Betancourt (1993), la tutoría se considera como un conjunto de actividades que propician situaciones de aprendizaje y apoyan el buen desarrollo del proceso académico con el fin de que los (acompañados) orientados y motivados desarrollen autónomamente su propio proceso.

Lo cierto es que, ya sean estas u otras las variantes de acompańamiento pedagógico que se escojan, se debe lograr la necesaria organización de las acciones que le son propias y que al final, definen las aportaciones del acompańamiento a la formación del docente, y con ella, al proceso de aprendizaje de los estudiantes. De ahí que se requiera, como precisa la UNESCO (1979), una política global para lograr que la educación del personal docente quede reorganizada como un proceso coordinado permanente, que empiece con la preparación previa al ejercicio de la profesión y que prosiga a todo lo largo de la vida profesional de los maestros y profesores. Esto significa que el acompañamiento pedagógico debe ser planificado en armonía total con el Plan de Superación y con el resto de planes y programas del centro educativo. 
El documento principal de todo el accionar docente-administrativo del centro educativo en la enseńanza primaria dominicana es el Proyecto Educativo de Centro (Plan de acciones contentivo de un sistema de operaciones técnico-administrativas coherentes con las necesidades y características específicas de cada institución educativa de este nivel de enseñanza). Dicho documento, que debe tener como soporte esencial la realidad de cada centro educativo, definida con el diagnóstico acertado de las fortalezas, oportunidades, debilidades y necesidades de la institución, debe incluir todo lo que en el orden de la formación didáctica debe realizar el centro educativo, con la finalidad de alcanzar la necesaria eficiencia del desempeño de los docentes. En ese Proyecto de Centro, en cuya concepción debe tomar parte toda la comunidad escolar (estudiantes, docentes, técnicos, administrativos, familia...), el acompañamiento pedagógico debe constituir una de las partes medulares dado el valor que tiene el desarrollo didáctico permanente, sistemático y puntual de las personas involucradas en el trabajo directo con los estudiantes.

El acompañamiento pedagógico es fundamental en el ámbito de la didáctica, como sistema de actividades que de forma permanente se ejecutan con y por los docentes, para garantizar las transformaciones dirigidas al desarrollo eficiente del proceso docente educativo y, en combinación con las diferentes formas de superación profesional; permite alcanzar la idoneidad del docente.

Ese sistema de acciones didáctico-metodológicas no es responsabilidad exclusiva del equipo de gestión de la escuela, sino que corresponde a todas aquellas personas que de uno u otro modo influyen en el proceso de enseñanza-aprendizaje. Es decir, es responsabilidad de los técnicos y especialistas de todos los niveles e instancias; pero de forma directa y especialmente, de los equipos de docentes de cada materia, grado, ciclo, área...

Las actividades de formación didáctica conducentes al desarrollo profesional del docente se deberían materializar en acciones concretas, donde el acompańamiento a la labor docente en el aula es fundamental; pero comprende, además: visitas y análisis de todas las actividades docentes y extradocentes, talleres y reuniones metodológicas, monitoreo de la preparación de la asignatura, clases metodológicas, clases demostrativas y clases abiertas, entre otras.

Estas tres últimas acciones son de las menos comunes en las escuelas primarias dominicanas y podrían ser objeto de un análisis por separado. En este, la pretensión es hacer referencia a la implementación eficiente de una de estas modalidades de formación didáctica, el acompañamiento pedagógico, en atención a que es la más común e integradora de todas, y transversal a las demás.

En la escuela primaria dominicana se proyecta el perfeccionamiento continuo de los acompañamientos. Asimismo, hay acciones y aportaciones teórico-prácticas de gran importancia, aunque sea a lo externo de la escuela. Una de ellas es el Diplomado en Acompańamiento Pedagógico que imparte el Instituto Tecnológico de Santo Domingo (INTEC) y otras instituciones de educación superior del país.

Como parte de ese interés por esta estrategia formativa del docente en el país, Castro y Ozoria (2007) aportan la tesis "Aplicación de las técnicas y estrategias de acompañamiento para el mejoramiento de la calidad de la enseńanza en la Escuela Divina Providencia". El documento tuvo como objetivo principal analizar las técnicas y estrategias de la supervisión en el mejoramiento de la calidad de la enseñanza en esa escuela básica del municipio Consuelo. Los análisis realizados indican que la escuela cuenta con un equipo de gestión que acompaña a los docentes y les ayuda a mejorar el proceso de enseñanza-aprendizaje. Según la investigación, la escuela Divina Providencia realiza el trabajo de acompańamiento de forma satisfactoria. El estudio recomienda supervisar, mediante un plan estratégico, la mejoría constante de la calidad de la enseñanza en los centros educativos. A 
los directores de los centros educativos, sugiere usar técnicas y estrategias adecuadas para lograr un aprendizaje de calidad.

El trabajo de acompañamiento encuentra fundamentación teórica en estudios de diversos intelectuales del área educativa. El maestro brasileño Paulo Freire (2010) hace referencia a la importancia de la formación permanente de los maestros. En tal sentido afirma que “...el momento fundamental en la formación permanente de los profesores es el de la reflexión crítica sobre la práctica” (p. 34); es decir, durante el ejercicio de su labor y en el intercambio con el resto del colectivo pedagógico.

Marcelo y Vaillant (2009) consideran que algunas de las características inherentes a los docentes:

(...) se refieren al conocimiento y los valores que maestros y profesores deben poseer para transmitir a los estudiantes, a los que se agrega el manejo de métodos de enseñanza relacionados con los contenidos, las competencias comunicacionales que les permitan interactuar con estudiantes, padres, colegas; el dominio de técnicas derivadas de los avances más modernos de las tecnologías de la información y la comunicación, las competencias para la investigación, y la reflexión acerca de sus propias prácticas. (p. 9)

Esto demanda que el maestro sea acompañado en la consecución de su labor pedagógica, siempre mediante acciones que estén en consonancia con las características, preparación y necesidades que le son propias. Así, es imprescindible que el acompañante evalúe cuáles características están presentes en cada docente de su radio de acción y que individualice su acción de acompañamiento.

El trabajo en colectivo, el apoyo mutuo, la cooperación, que en su esencia son sinónimos del acompańamiento, ayudan a la materialización de un desempeño eficiente de la labor del maestro en su aula. Al respecto, Fullan y Hargreaves (2006) expresan que en las escuelas eficaces, como observa Rosenholtz, el trabajo en equipo se asocia a normas y oportunidades que dan sustento a la mejora sostenida y al aprendizaje permanente (en toda la carrera): "El supuesto es que la mejora de la enseñanza constituye una empresa más colectiva que individual, y que el análisis, la evaluación y la experimentación en compañía de sus colegas son condiciones bajo las cuales el docente mejora” (p. 82).

Cuando la ayuda que se brinda al docente es una farsa, una simple estratagema para aparentar la labor metodológica que corresponde, o cuando esa ayuda es arropada por el papeleo y la burocracia, nunca se obtendrán resultados reales. Freinet, citado por Bixio (2005) afirma que "el peor error de la escuela tradicional es el de desanimar al personal docente, desviando y reprimiendo las iniciativas renovadoras en aras a una serie de fundamentos y premisas que no pasan de ser meros criterios burocráticos" (p. 119).

El trabajo de acompañamiento pedagógico, con todo lo que él incluye, no es un término de nuestros días ni de un sistema de educación específico; es un concepto de necesaria recurrencia en los diferentes ámbitos educativos que apuestan por la eficiencia en la formación de las nuevas generaciones de educadores. En tal sentido, acerca del desarrollo profesional de los docentes en el ámbito dominicano, es pertinente mencionar las aportaciones de Eugenio María de Hostos.

Almánzar (2008) afirma que:

Hostos estructuró la Escuela Normal en dos secciones: una práctica y otra teórica. La primera servía de preparación para la segunda y abarca la enseńanza primaria: la segunda estaba destinada a los estudios formativos que debían cursar los futuros Maestros Normales; quienes también debían realizar prácticas pedagógicas, como un medio eficaz para adquirir las destrezas necesarias para transmitir con eficiencia y capacidad el proceso de enseñanza-aprendizaje. (p. 55) 
Por supuesto que, la referida práctica pedagógica tiene como componente fundamental el acompańamiento del docente; es decir, la demostración, el diálogo, el intercambio y el aprendizaje compartido.

A propósito de la labor de Hostos, en nuestros días, el MINERD (2010), señala:

La realización de toda actividad metodológica está encaminada a que los cuadros, funcionarios y el personal docente graduado y en formación, se prepare (...) y domine los contenidos, la metodología del trabajo educativo, la didáctica de las asignaturas, especialidades o áreas de desarrollo que imparten con un enfoque científico y sobre la base de satisfacer las exigencias siguientes: (a) Elevar la calidad del proceso educativo mediante el perfeccionamiento constante de su labor profesional. (b) Lograr la preparación en la práctica, de manera sistémica y sistemática, de todos los dirigentes (...) docentes graduados y en formación, así como de los técnicos. (c) Perfeccionar el desempeño profesional científico y creativo sobre la base de actuaciones éticas en correspondencia con la tradición pedagógica (p. 47).

Al ser el acompañamiento pedagógico una variante para la formación didáctica del docente, hay que tomar en cuenta estas exigencias cada vez que se realice una acción de acompañamiento. Si no se cumple con ellas, quedará un vacío, una falta de coherencia entre la acción y los resultados que se procuran.

Desde diferentes estudios y escenarios, con frecuencia se revela hacia dónde debe enfilarse la formación didáctica en la escuela primaria dominicana. El Ministerio de Educación (MINERD) y el Ministerio de Educación Superior, Ciencia y Tecnología (MESCyT) de forma sistemática declaran aquellas áreas donde hay que hacer mayor énfasis en los acompañamientos y demás acciones metodológicas. El MESCyT (2012) asegura que:

“...los docentes no manejan adecuadamente la cuestión curricular y que en sus prácticas pedagógicas en el aula muestran un pobre desempeńo, por lo que existe la necesidad de que se promueva el reforzamiento de las competencias para "comunicar, manejar la incertidumbre y la inseguridad generada por un mundo donde el conocimiento se renueva permanentemente". (p. 57)

El citado estudio, liderado por el MESCyT, el cual tenía la intención de establecer un programa de reformulación de la formación docente (p. 22), da cuenta de que no se desenvuelve al ritmo deseado y que tampoco se alcanzan los resultados previstos en materia de formación. Incluso, se precisa que existe una necesidad impostergable de establecer estándares que funcionen como requerimientos a ser cumplidos por las instituciones formadoras de docentes (p. 23). Por esto, en el año 2014 dichos estándares fueron establecidos (MINERD, 2014).

Asimismo, el Modelo de gestión de la calidad para los centros educativos (SEE, 2004) establece, entre otros, el llamado "Principio de la participación", el cual declara que "La participación de todos los miembros de la comunidad educativa es la mejor garantía para el desarrollo de los compromisos y asegurar la continuidad de los procesos de transformación" (p. 25).

\section{Método}

La investigación que sirvió de base al presente artículo tuvo la intención de determinar la percepción de los docentes acerca de la organización del acompañamiento pedagógico en dos centros 
educativos de Santo Domingo, Distrito Nacional. Para lograrlo, se procedió a constatar la opinión de los docentes acerca de la organización del acompañamiento a nivel de centro. Se buscaba defender la idea de que la organización del acompañamiento pedagógico a nivel de centro, no responde a las necesidades de desarrollo profesional específicas de los docentes.

El estudio se realizó en un centro educativo público (escuela) y un centro educativo privado del Distrito Escolar 15-03. Ambos, centros urbanos, de amplia trayectoria por sus años de servicio y con cuerpos docentes calificados, de vasta experiencia y prestigio social. Los estudiantes del centro educativo proceden de familias de clases media y alta; mientras, los estudiantes de la escuela proceden, esencialmente, de familias de clase baja. La selección de la muestra se hizo por cuotas ya que se requería una representación de ambos ciclos ( $1^{\circ}$ a $3 e r$ grados y $4^{\circ}$ a $6^{\circ}$ grados, respectivamente) del Nivel Primario. De cada uno de los centros se seleccionaron 10 maestros, un coordinador y un director.

La investigación asumió un enfoque cualitativo ya que la definición de preguntas se realizó en el mismo proceso de recolección de datos; es decir, de manera dinámica entre los hechos y su interpretación. Fue una investigación descriptiva, ya que se detalla un fenómeno social en una circunstancia temporal y geográfica determinadas. Así, se empleó el método cualitativo o no tradicional, según clasificación de Bonilla y Rodríguez (Bernal, 2010). Fue seleccionado éste ya que la intención era profundizar en casos específicos, no generalizar; la prioridad fue cualificar y describir el fenómeno objeto de estudio a partir de rasgos determinantes, según fueran percibidos por los sujetos que están dentro de la situación estudiada. Fue empleado el proceso inductivo-deductivo por tener su base en la lógica y ser útil en el estudio de hechos particulares.

En correspondencia con el método elegido, se optó por diferentes técnicas de obtención de información: entrevista no estructurada, grupo focal y análisis documental. Como el problema estudiado es subjetivo (la percepción de los docentes), fue de gran utilidad el desarrollo de este tipo entrevistas dada su flexibilidad: el entrevistador definió la profundidad del contenido, la cantidad y el orden de las preguntas y cuestiones a tratar con el entrevistado, con la marcada intención de acceder a una información específica. Las entrevistas a directivos y técnicos de ambos centros educativos siguieron el mismo protocolo y cuestionario. Estas entrevistas fueron desarrolladas en dos grupos específicos de sujetos: los maestros del nivel primario y el formado por los directivos y técnicos de la escuela, sin variación en los tópicos empleados. En ambos casos se emplearon preguntas generales que, a partir de una guía de contenido, sin categorías preestablecidas, permitieron la expresión libre de experiencias personales, sin ningún tipo de influencia por parte del entrevistador.

El grupo focal permitió conocer cómo los participantes se forman una perspectiva acerca del problema estudiado, a través de la interacción. Las sesiones de trabajo se desarrollaron con dos grupos focales donde los participantes expresaron libremente su opinión sobre los aspectos relacionados con el acompańamiento pedagógico dentro de la organización de la formación docente continua a nivel de centro.

La grabación que se empleó, tanto de las entrevistas como de las sesiones de grupos focales fue total, lo cual permitió el estudio detallado de cada respuesta y comentario de los participantes. Esto facilitó la contrastación de respuestas para buscar coherencias y contradicciones entre las mismas.

Mientras, el análisis documental sirvió para la recopilación y estudio de documentos de los centros unidades de estudio relacionados con el acompañamiento y la formación continua de los docentes. La revisión y análisis de documentos permitieron verificar las acciones que la escuela prevé, específicamente en el área de desarrollo profesional de los docentes, y su coincidencia con las 
necesidades formativas de su colectivo pedagógico. La citada revisión permitió, asimismo, observar la sistematicidad de esas acciones.

La triangulación metodológica de los resultados encontrados con cada técnica permitió obtener resultados más fiables para la explicación del problema de investigación. Esta triangulación metodológica hizo énfasis, más que en lo conceptual, en la percepción de los docentes acerca de la organización de las acciones de desarrollo profesional que realiza la escuela.

El análisis de los datos, basado en diagramas y mapas conceptuales, se realizó a partir de la interpretación y valoración de los hechos y las informaciones obtenidas en el estudio, expresadas mediante la narración.

\section{Resultados}

La búsqueda, mediante la aplicación de las técnicas y los instrumentos previstos para cada una, permitió descubrir que los directivos y técnicos en ambos centros educativos consideran que los maestros participan en la organización de los procesos de acompañamiento del centro educativo. Estos afirman que las necesidades docente-metodológicas de los maestros definen qué acciones deben realizarse. A la vez, ven como propias del acompañamiento en la escuela, las actividades que realizan otras instituciones. Es decir, no conciben el trabajo de acompańamiento como centro de la labor de la escuela y su equipo de gestión, sino como aquellas acciones que coordinan con especialistas de otras instituciones. Desde su perspectiva, la escuela cumple con la encomienda que tiene en el proceso de desarrollo profesional de los docentes y las actividades que ejecutan tienen la calidad que requiere ese tipo de tarea.

Fue posible constatar cómo se realiza la organización de las acciones de formación docente en la escuela, mediante la observación del documento Proyecto Educativo de Centro (PEC) de cada una de las unidades de estudio, las entrevistas y grupos focales, como se muestra a continuación.

\section{Tabla 1}

Matriz: Organización de la formación docente

\begin{tabular}{|c|c|c|c|}
\hline Indicadores & Observación & Entrevistas & Grupo focal \\
\hline $\begin{array}{l}\text { Organización de las acciones de } \\
\text { desarrollo profesional docente. }\end{array}$ & $\begin{array}{l}\text { Las acciones previstas en el } \\
\text { Proyecto Educativo de Centro } \\
\text { (PEC) son mínimas en cantidad. } \\
\text { No se precisa cada una de las eta- } \\
\text { pas y acciones concretas. El traba- } \\
\text { jo no es sistemático ni responde a } \\
\text { las necesidades particulares de los } \\
\text { maestros. Son planificadas como } \\
\text { acciones propias de la escuela, los } \\
\text { talleres en un tema determinado } \\
\text { y que ejecutan especialistas e ins- } \\
\text { tituciones externas. }\end{array}$ & $\begin{array}{l}\text { Los directivos expresan que, al } \\
\text { organizar las acciones formati- } \\
\text { vas en el PEC, toman en cuenta } \\
\text { las necesidades de su colectivo, } \\
\text { a partir de un diagnóstico que } \\
\text { se realiza a inicios de curso. Los } \\
\text { maestros desconocen dónde, } \\
\text { cuándo y cómo son definidas y } \\
\text { planificadas las acciones formati- } \\
\text { vas de su escuela. Ellos las definen } \\
\text { como "talleres y seminarios". }\end{array}$ & $\begin{array}{l}\text { Los participantes aseguran que } \\
\text { "son los directivos quienes defi- } \\
\text { nen lo que se hace. Desconocen } \\
\text { a partir de qué criterios se realiza } \\
\text { la organización y planificación de } \\
\text { las acciones de superación en que } \\
\text { participan. Coinciden en que du- } \\
\text { rante el curso se planifican alrede- } \\
\text { dor de tres a cuatro actividades de } \\
\text { superación. }\end{array}$ \\
\hline
\end{tabular}




\begin{tabular}{|c|c|c|c|}
\hline Indicadores & Observación & Entrevistas & Grupo focal \\
\hline $\begin{array}{l}\text { Percepción de los docentes acerca } \\
\text { de la organización de las acciones } \\
\text { de formación docente }\end{array}$ & No aplica. & $\begin{array}{l}\text { Los directivos y técnicos de ambas } \\
\text { unidades de estudio dicen que los } \\
\text { maestros participan en la organiza- } \\
\text { ción de las acciones de desarrollo } \\
\text { profesional docente. Afirman que las } \\
\text { necesidades de los maestros definen } \\
\text { qué acciones de "capacitación" deben } \\
\text { realizarse. Ven como propias de la es- } \\
\text { cuela las actividades que realizan otras } \\
\text { instituciones en las que participan sus } \\
\text { maestros. } \\
\text { Los docentes revelan que la plani- } \\
\text { ficación es vertical: las autoridades } \\
\text { deciden qué acciones metodológicas } \\
\text { deben ser desarrolladas en cada etapa } \\
\text { del ańo escolar. }\end{array}$ & $\begin{array}{l}\text { Los maestros precisan que no partici- } \\
\text { pan en la organización de las acciones } \\
\text { formativas que realiza la escuela. Sus } \\
\text { principales necesidades de formación } \\
\text { no son determinantes a la hora de } \\
\text { planificar las acciones formativas en } \\
\text { la escuela. Ven como parte de estas } \\
\text { el apoyo económico que reciben los } \\
\text { interesados en participar en diploma- } \\
\text { dos, maestrías, eventos... Expresan } \\
\text { que las actividades formativas de la } \\
\text { escuela no se corresponden con sus } \\
\text { necesidades. }\end{array}$ \\
\hline
\end{tabular}

Fuente: Elaboración propia a partir de la investigación (Galán, 2014)

Es significativo que más el $90 \%$ de los maestros entrevistados y los participantes en los grupos focales aseguran que no toman parte en la organización de las acciones metodológicas que realiza la escuela. Asimismo, el 70\% señalaron que sus principales necesidades para el desempeño de su labor docente no son determinantes a la hora de planificar las acciones de superación en la escuela. Ven como parte del acompañamiento el apoyo económico que reciben los interesados en hacer diplomados, maestrías; participar en evento. Cinco de los 10 entrevistados expresaron que las actividades que la escuela emprende no siempre se corresponden con sus necesidades personales.

Entre la opinión de los maestros entrevistados y la de los que participan en los grupos focales hay significativas coincidencias en cuanto a su nivel de participación en la organización del acompañamiento a nivel de escuela. Así, la percepción que tienen los equipos de gestión institucional al respecto es opuesta a la de los maestros: para los primeros, lo que hace la institución es lo correcto, aun cuando sus propios miembros reconocen la pobre cantidad de tareas concretas y la falta de diversidad en las mismas; y cuando revelan que los maestros no participan en la definición de cuáles actividades, y cuándo y cómo se realizan. Los docentes hacen referencia a cuál es su rol en esas tareas: receptores de información y asistentes pasivos a talleres y seminarios. Además, es un proceso, según los propios entrevistados, que tiene poca descentralización y sí un palpable verticalismo: desde las instancias superiores no existen orientaciones y acciones precisas conducentes a que el centro educativo ejecute un trabajo que parta de una organización precisa, en la que participen todos los miembros de la comunidad educativa y en la que se prevea el empleo de una diversidad de acciones que faciliten la atención a la diversidad y a las necesidades particulares de los docentes.

El acompańamiento en las unidades de estudio fue descalificado por el $80 \%$ de los maestros que participaron en el estudio ya que, además de limitar su participación en la organización, no cumple con su etapa básica: el diagnóstico. En los centros educativos estudiados, se revela que el diagnóstico se limita a consideraciones y evidencias superfluas que no aportan los elementos necesarios para determinar qué acciones son las pertinentes. Sin embargo, este debería ser profundo e individual, un estudio del colectivo docente para determinar la ayuda concreta, la tarea precisa a acometer en correspondencia con las necesidades individuales. 
Es palpable que no se asume el acompañamiento pedagógico como la estrategia de la escuela para propiciar la creatividad individual y colectiva, y el desarrollo profesional de los maestros. Asimismo, los actores expresan que desde la escuela no se otorga el valor que tiene la participación de todos los docentes en la organización de las acciones de desarrollo profesional, cómo vía de preparación para la conducción del aprendizaje. Dichas acciones se limitan a la ejecución de talleres, seminarios y escasas visitas a las tareas docentes dentro y fuera del aula. Mientras los miembros de los equipos de gestión defienden la calidad y cantidad de actividades de superación que se realizan en la escuela, los maestros las consideran insuficientes y no observan mayores beneficios en las mismas.

En las diferentes sesiones de trabajo con uno de los grupos focales, en cada uno de los cuales toman parte seis maestros, estos comparten los mismos criterios en cuanto al concepto acciones de desarrollo profesional. Para ellos, estas no son más que los talleres que realizan determinadas instituciones en coordinación con los centros educativos. Una de las participantes asegura que: “...no es más que el seguimiento que se le da al maestro desde su área, que da el refuerzo que le hace falta, el apoyo, estrategias, cambios...”. En el otro grupo focal, los maestros ven estas como la labor que el equipo de dirección y técnico realiza, durante el inicio y después del primer semestre del curso, con la intención de desarrollar temas de interés general que deberán ser tomados en cuenta en su labor docente. Otros asistentes a un grupo focal aseguran que son los miembros de los equipos de gestión quienes determinan qué se hará en un encuentro formativo y cómo.

En la planificación que hacen las escuelas, sobre todo en el Plan Educativo de Centro (PEC), no hay una definición conceptual ni organizativa de las actividades que corresponden al acompañamiento pedagógico y a la formación docente continua. La realización de las escasas acciones que se incluyen en los documentos, no es prevista desde las posibilidades objetivas del centro educativo, sino desde las propias de instituciones y personas externas que les colaboran.

Los directores y coordinadores entrevistados aseguran que los docentes toman parte en la organización de la formación de los docentes. Precisan que una muestra es que "se toman en cuenta las necesidades de los maestros", "se parte de un diagnóstico de los docentes para definir las actividades de superación a realizar". Los directivos entrevistados señalan como acciones de carácter didáctico aquellas que se realizan en la escuela por parte de instituciones y especialistas externos. Los talleres y seminarios son las modalidades que se exponen como esencias del trabajo de desarrollo profesional.

Cuatro de los maestros entrevistados afirman, categóricamente, que ellos no toman parte en esa organización, sino que es función de la dirección de la escuela determinar qué actividades se realizan.

Las actividades de acompańamiento desde la escuela no son vistas por los maestros como algo específicamente formativo. Uno de los entrevistados lo ejemplifica de este modo: "En seis años que llevo aquí, me han visitado entre 3 y 4 veces, pero después no hay un seguimiento".

Ninguno de los maestros expresa tener conocimiento de las acciones previstas en la escuela para la formación docente durante el curso escolar, ni de si están o no plasmadas en las planificaciones del centro educativo.

Entre la opinión de los maestros entrevistados y la de los que participan en los grupos focales hay significativas coincidencias en cuanto a su nivel de participación en la organización de las acciones formativas a nivel de escuela. En las sesiones de grupo focal organizadas, los participantes aseguran que "no toman parte en la organización de la superación". Aseguran que "son los directivos quienes definen qué acciones docente-metodológicas se realizan y quiénes deben tomar parte en ellas". 


\section{Discusión y conclusiones}

A través de las técnicas (observación de documentos, entrevistas y grupo focal) empleadas para buscar respuesta al problema definido en esta investigación, se ha llegado a elementos de gran importancia en la organización de las acciones de desarrollo profesional del docente de primaria. Un análisis detallado de los resultados obtenidos revela que no hay coherencia entre las formas de organización del trabajo de formación didáctica en las unidades de estudio y lo que realmente debería acontecer en la escuela. Si se sigue un orden lógico para dicho análisis, la acción que debe marcar el inicio de ese trabajo es un diagnóstico profundo e individual, un estudio de las necesidades formativas del colectivo docente para determinar la ayuda concreta, la tarea de desarrollo profesional precisa a acometer. En las unidades de estudio de la investigación base de este texto, el diagnóstico se limita a consideraciones y evidencias superfluas que no aportan los elementos necesarios como para determinar qué acciones formativas son las adecuadas.

Los resultados obtenidos permiten asegurar que el docente no participa en la definición y organización de las acciones de desarrollo profesional que se conciben en el centro educativo. Mientras, la cohesión que debe existir en este trabajo se ve afectada al no tomar en cuenta la unidad interna de los componentes que deberían definirlo: personal, organizativo y funcional. Resulta palpable que no hay diversidad de actividades de formación en las unidades de estudio ni van dirigidas a la formación didáctica concreta, específica y práctica.

Aun cuando es conocido que las acciones de formación continua deben ser previstas en el Plan Educativo de Centro, en los centros educativos objeto del estudio, este recurso de organización del trabajo, no refleja una clara definición de tareas didácticas dirigidas a la formación de sus maestros y a proyectarlos hacia un desempeño eficiente de su labor. En resumen es perceptible la necesidad de un trabajo de formación didáctica integradora, sistemática y continua.

La práctica educativa en la institución escolar está condicionada por el nivel de organización que se logre en todas las tareas concernientes a la labor pedagógica. Casi siempre están en equilibrio los niveles de eficiencia de la práctica educativa con la eficiencia lograda en la planificación de todas las acciones técnico-metodológicas del centro educativo, esencialmente el acompañamiento. Cuando no se conciben, planifican y realizan las labores que demanda el colectivo pedagógico, habrán sido desaprovechados el tiempo, los esfuerzos y los recursos.

Como la calidad de la práctica educativa depende de la eficiencia de la planificación, esta última debe ser vista como núcleo de la gestión en cada centro docente. En una verdadera planificación hay cuatro momentos de un ciclo que se repiten de manera periódica y coherente: fijación de metas y objetivos, diagnóstico, programación, evaluación (Barone, 2007, 53).

Ese ciclo propio de la planificación para todo el proceso de gestión de un centro escolar es aplicable a la planificación de las acciones formativas en el orden didáctico, y dentro, de ellas el acompañamiento pedagógico. Todas las tareas que se definan en función del desarrollo profesional de los docentes deben cumplir con estas etapas y en cada una de ellas el propio docente debe ser un real protagonista. 


\subsection{Metas y objetivos}

La determinación de los objetivos a lograr en el acompañamiento no es una responsabilidad exclusiva del equipo de gestión del centro educativo; tampoco es una responsabilidad de los docentes acompañantes, los técnicos y demás especialistas externos que influyen en el centro educativo. El equipo de gestión tiene la responsabilidad de conducir la participación activa de todos los miembros del colectivo pedagógico en la precisión de qué hacer y con qué propósito; es decir, no cabe improvisación ni decisión unilateral: todos son corresponsables de qué acciones de desarrollo realizar y cómo.

Al precisar los objetivos de los acompańamientos sería acertado tener en cuenta a qué docentes van dirigidos y cuáles son las particularidades de su formación y desempeño. Es oportuno tener considerar los criterios de Imbernón (1994), quien analiza el desarrollo profesional del profesorado en dos etapas:

1. El desarrollo profesional de los profesores noveles (la etapa de inducción o socialización a la profesión).

2. El desarrollo del profesorado experimentado, destacando, además, tres grandes líneas en la formación permanente de profesores: La reflexión sobre la propia práctica (mediante el análisis de la realidad educativa) y la comprensión, interpretación e intervención sobre ella; el intercambio de experiencias, la necesaria actualización y confrontación en todos los campos de la intervención educativa; y el desarrollo profesional, en y para el centro, mediante el trabajo colaborativo para transformar esa práctica y provocar procesos de comunicación (p. 86).

No existe manera más precisa de lograr el mencionado trabajo colaborativo que con un verdadero proceso de acompañamiento continuo, puntual y creciente en dinámicas, herramientas y recursos que respondan a necesidades e intereses.

Este mismo autor propone cinco modelos para la formación permanente del profesorado ( $\mathrm{pp}$. 12-17):

- El modelo de formación orientada individualmente

- El modelo de observación/evaluación

- Modelo de desarrollo y mejora

- Modelo de entrenamiento o institucional

- Modelo de investigación o indagativo

Todos estos modelos son de gran valor en el acompañamiento pedagógico, mediante el cual se procura la formación, el seguimiento, el desarrollo, así como el entrenamiento y la participación en acciones de carácter científico-investigativo.

\subsection{Diagnóstico}

Las acciones de acompañamiento pedagógico, por su carácter de sistema, deben partir del diagnóstico preciso e individual. Esta evaluación de entrada permite definir hacia dónde deben dirigirse las actividades y las acciones que le son propias, y cerrar con el control para repetir un nuevo ciclo en el que se convierten en medulares la preparación, la observación, la orientación y la demostración; pero sobre todo el intercambio, mediante la indagación dialógica.

El diagnóstico no es un momento específico de la práctica educativa, ni un apéndice de su planificación. El diagnóstico debe estar presente en todas y cada una de las acciones; pero, además, debe 
estar dirigido a las personas que participan, a las acciones que se ejecutan y a los resultados que se alcanzan en cada etapa. Otro tanto debe suceder con las acciones de evaluación de las actividades: sistemáticas, individuales, coherentes con los objetivos a lograr... Dicha evaluación, en la propia dinámica del diagnóstico, debe ser continua, sistemática e individual.

\subsection{Programación}

El diseño de un programa para el trabajo de desarrollo profesional del docente es una tarea obligatoria y deberá estar articulada al Plan de Formación, donde el docente asume tareas de desarrollo personal como parte de su autoformación. Esto equivale a decir que los acompańamientos pedagógicos no deben ser improvisados; deben ser previstos con antelación e incluir actividades en correspondencia con las necesidades individuales del docente, las cuales deben ser resultado del diagnóstico inicial y continuo. En correspondencia con diversos factores se determina la ejecución de una u otra acción de acompañamiento y, como es lógico a su programación, podrían formularse preguntas como las siguientes:

- ¿A quién acompañar?

- ¿Quiénes más participarán en el acompañamiento?

- ¿Por qué acompañar a determinado docente, grupo escolar, materia...?

- ¿Cuándo hacer el acompañamiento (etapa del año escolar, día, hora)?

- ¿Qué recursos son necesarios para la ejecución del acompańamiento?

Estas y otras interrogantes ofrecerán las razones previas para la acertada planificación y el desarrollo eficiente del acompańamiento. Entre ellas debe ocupar el centro la intención de mejorar los aprendizajes de todos y cada uno de los estudiantes.

\subsection{Evaluación}

La evaluación del acompañamiento cierra un ciclo que debe repetirse de forma sistemática. A partir de la valoración y los resultados concretos que se alcanzan en una actividad, se prevén las demás acciones de acompañamiento a implementar. En dicha evaluación deben tomar parte todos los participantes. No se debe perder de vista que el acompańamiento tiene como finalidad que el personal docente desarrolle con eficiencia su labor en el proceso de enseńanza-aprendizaje. Para lograrlo, cada acompańamiento tendrá como centro el desarrollo profesional de los docentes para el logro de la eficiencia en su labor, para la enseńanza de las materias que atiende, el trabajo práctico y de laboratorios, el estudio de la metodología a emplear en la formación integral y el conocimiento de las particularidades de los estudiantes.

Por eso el acompańamiento pedagógico en la enseńanza primaria debe ser sistemático, profundo y coherente; debe tener como atributos el debate y la atención a la individualidad. Asimismo, debe estar encaminado a elevar el nivel científico-teórico y pedagógico-didáctico del colectivo escolar, como factor indispensable para el desarrollo integral del docente. A propósito, podrían establecerse determinadas etapas del acompańamiento pedagógico, todas ellas con la necesaria flexibilidad, coherencia y posible adaptación a la realidad de los actores, sus intereses, motivaciones y competencias. 


\subsection{Etapas del Acompańamiento Pedagógico}

Los momentos del ciclo definido por Barone (2007) son coherentes con cuatro etapas básicas del acompañamiento pedagógico. Para que éste constituya un método efectivo en la elevación de la calidad del proceso de enseñanza-aprendizaje, es preciso definir una secuencia lógica y a la vez flexible, que se pueda acomodar a la realidad de cada centro educativo. En ese proceso continuo de preparación metodológica del docente, se debería procurar el cumplimiento de estas etapas:

Primera etapa: Preparación para el acompańamiento

Segunda etapa: Desarrollo del acompañamiento

Tercera etapa: Análisis de la actividad del docente

Cuarta etapa: Análisis del acompañamiento

\subsubsection{Primera etapa: Preparación del acompańamiento}

Esta etapa tiene como punto de partida el diagnóstico preciso del escenario en que se ejecutará la actividad. Incluye la coordinación de fecha y espacio, la determinación por el docente acompañante del docente y la actividad a compartir. El docente acompañante realiza una exploración acerca de la preparación de la actividad, la realidad del grupo y las competencias del docente para la tarea a desarrollar. Este es el momento para un diálogo con él y con los demás actores a incluir, como es el equipo de gestión de la escuela, para acceder al conocimiento que tiene acerca de las características del grupo y de cada estudiante, así como se debate entre los miembros del equipo de gestión acerca del docente y su labor. El acompañante deberá evaluar la calidad de la actividad prevista, los recursos necesarios, las acciones evaluativas a realizar, etc. De ese modo, podrá darle sugerencias, ayudarlo a concebir actividades y recursos, sugerirle formas de organización del grupo. Desde esta etapa del acompańamiento deben participar, además del acompañante, sobre todo el coordinador, el subdirector y otros docentes que pueden sumarse y aportar sugerencias y recomendaciones que hagan más rica la actividad. La preparación del acompañamiento se realiza antes de la participación en la actividad acordada con el docente; lo ideal es que se haga con suficiente tiempo de antelación, por ejemplo, una semana antes de su ejecución.

Para que lo anterior sea una realidad, el trabajo debe contener ciertos atributos que se pueden resumirse en:

- Hay precisión en su planificación, ejecución y evaluación.

- Es una vía de diagnóstico y seguimiento de las competencias de desempeño del colectivo pedagógico.

- Es esencialmente una actividad colectiva, donde participan los miembros del colectivo en todas sus etapas.

- Constituye una herramienta de preparación tanto individual como colectiva.

- Es un sistema de acciones coherentes donde prima la demostración.

- Define espacios concretos para el desarrollo de la acción científica.

- Es un espacio abierto a la participación de toda la comunidad escolar.

- Debe ser una actividad evaluable tanto en su proceso como por los resultados. 


\subsubsection{Segunda etapa: Desarrollo del acompañamiento}

Este es el momento en que se participa en el desarrollo de la actividad y se privilegia la indagación dialógica. El docente acompañante se mantiene atento a la labor del docente y de los estudiantes, hace las anotaciones pertinentes para luego emplearlas en el análisis. El visitante no interrumpe la actividad ni hace intervenciones que pongan en duda, frente a los estudiantes, las competencias del docente si éste se equivoca en una actividad, al final se hacen las correcciones pertinentes. El acompañante estará atento al desempeño de cada estudiante y a la atención individualizada que presta el docente. Es importante tener en cuenta el uso racional del tiempo (anotar el tiempo que se dedica a cada parte de la actividad); de capital importancia es la atención que presta a los estudiantes de acuerdo a la actividad que ejecutan. Aun cuando la actividad sea excelente, el acompañante debe buscar ese elemento en el cual vale aportar sugerencias para el perfeccionamiento del trabajo y el desarrollo de actividades similares.

\subsubsection{Tercera etapa: Análisis de la actividad del docente}

Después de realizada la visita de acompañamiento, los participantes realizan el análisis de la actividad y los resultados alcanzados en ella. Se realiza un diálogo reflexivo acerca de las fortalezas y debilidades observadas. En el análisis pueden participar otros docentes que serán beneficiados con las valoraciones y conclusiones a que se arribe. El punto centro del debate debe ser el objetivo previsto para la actividad. Lo fundamental es determinar hasta qué nivel los estudiantes (todos y cada uno) han llegado al logro del propósito. De aquí, ha de partir el análisis de cuáles fueron los factores que influyeron positiva y negativamente en que la actividad cumpliera o no lo previsto; por ejemplo, si el objetivo era escribir oraciones y resultó que la mayoría de los estudiantes no lo logró, esto indica que no se cumplió el objetivo y que determinados factores lo provocaron. La conclusión se desprende del diálogo basado en interrogantes como estas: ¿La actividad se desarrolló como fue prevista? ¿Los recursos se emplearon con la eficiencia que demandaba la actividad? ¿Fue atendida la diversidad de estudiantes? ¿Se aprovechó el tiempo racionalmente?, por solo citar algunos.

Para tener elementos más convincentes en el análisis, es importante apelar a la actividad que hicieron los estudiantes, sobre todo a la producción escrita en su cuaderno, y su corrección, si es el caso, o a las competencias que mostraron en su práctica de aprendizaje. De este análisis se derivan las recomendaciones y se precisan los acuerdos, vías de cumplimiento, recursos a emplear y fechas de control de los referidos acuerdos. Son importantes las modelaciones en dependencia de las necesidades del docente y la actividad acompañada.

\subsubsection{Cuarta etapa: Análisis del acompańamiento}

Durante esta etapa se realiza la retroalimentación del proceso de acompañamiento. El acompañado expone su opinión, los beneficios que ha obtenido de la actividad, los elementos que considera deben tenerse en cuenta para próximas actividades similares; sugiere en qué otra u otras actividades le gustaría ser acompañado. Los demás asistentes exponen sus puntos de vista, sus críticas a la actividad y sus propuestas de mejoras. Es el momento para que el docente acompañante reflexione 
críticamente acerca de los aciertos y fallas en la actividad y para replantear acciones futuras similares. Este proceso deberá ocurrir en un dinámico intercambio en el que todos sean protagonistas y donde se manifieste un ambiente de camaradería, respeto y ayuda mutua.

Es necesario subrayar el valor que tienen la creación de un ambiente adecuado de trabajo en todo el proceso de acompańamiento pedagógico, la coordinación entre los miembros de los equipos de gestión de los centros educativos, el tomar en cuenta los intereses, recursos, disposición y la preparación para esta actividad. Singular importancia tiene también el conocimiento de la situación real y específica de cada contexto, centro, equipo de gestión, docente, grupo, área y estudiante. Es medular el seguimiento a los acuerdos anteriores, a las situaciones detectadas con antelación. De ahí que sea necesario el control y seguimiento sistemático del trabajo.

Es tarea de cada miembro del equipo de gestión, la determinación de estrategias de trabajo dirigido a la formación didáctica, que no se limiten al acompañamiento pedagógico, sino que lo complementen, que tengan como esencia la implementación de actividades, fundamentadas científica y didácticamente, y de utilidad para los actores y espacios. Entre dichas estrategias se pueden privilegiar las clases abiertas, metodológicas y demostrativas; la preparación de las actividades concretas de la asignatura y el área, con las más flexibles variantes para su organización, ejecución y control.

Estos resultados obtenidos en el estudio, junto con la experiencia personal desarrollada en varios ańos de labor como formador y acompañante de docentes, nos permiten afirmar que existe la necesidad de acciones científico-metodológicas que estimulen la participación activa de los miembros de las comunidades educativas. Dicha participación debería ser desde su organización en el centro educativo como vía a favorecer un desempeño profesional eficaz del docente y del resto de los involucrados en la búsqueda de la calidad de la educación. Ninguna otra estrategia permite dichas acciones como el acompañamiento pedagógico, pero sólo si su organización es coherente con la realidad del contexto y de cada uno de los actores.

Es pertinente el desarrollo de encuentros de seguimiento, retroalimentación y debate científico acerca del proceso de organización y desarrollo del acompańamiento pedagógico, y de otras variantes de preparación del docente que se revelen como un verdadero sistema de formación continua.

\section{Reconocimientos}

Agradecemos a Ancell Scheker por facilitarnos la base de datos, y por permitir al CILGE introducir instrumentos para medir características de liderazgo y gestión de los directores en el protocolo de aplicación de la Evaluación Diagnóstica del Primer Ciclo de Educación Media, 2013. También agradecemos a la Fundación INICIA por el financiamiento y apoyo en la elaboración de este artículo, y seguir desarrollando algunas de las líneas de investigación del CILGE. Además, esta investigación se ha beneficiado del intercambio académico de los participantes del Congreso Internacional del IDEICE 2012 y de los comentarios de los participantes de los Workshops organizados por el CILGE para el MINERD, durante el 2012, 2013 y 2014. 


\section{Referencias}

Aguerrondo, I. y Xifra, S. (2002). La escuela del futuro I. ¿Cómo piensan las escuelas que innovan? Buenos Aires: Papers Editores.

Aguerrondo, I.; Lugo, M. T.; Rossi, M.; Pogré, P. y Xifra, S. (2002). La escuela del futuro II. ¿Cómo planifican las escuelas que innovan? Buenos Aires: Papers Editores.

Aguerrondo, I.; Lugo, M. T.; Rossi, M. \& Tedei, P. (2002). La escuela del futuro III. ¿Qué hacen las escuelas que innovan? Buenos Aires: Papers Editores.

Almánzar García, J. N. (2008). Trayectoria de la formación del docente dominicano. Desde 1880 hasta el año 2000. Santo Domingo: SEE.

Bigge, M. I. (1979). Teorias de aprendizaje para maestros. Ciudad de México: Editorial Trillas.

Fullan, M. \& Hargreaves, A. (2006). La escuela que queremos. Los objetivos por los cuales vale la pena luchar. Buenos Aires: Amorrortu Editores.

Imbernón, F. (1994). La formación y desarrollo profesional del profesorado. Hacia una nueva cultura profesional. Barcelona: Editorial Graó.

Jacobo García, H. M. (coord.) (2005). Acompañamiento sistémico: lo que los educadores podemos hacer en contexto. Cuadernos de investigación: educación de migrantes. México: Secretaría de Educación Pública y Cultura del Gobierno de Sinaloa.

Labarrere Sarduy, A. F. (2008). Bases conceptuales de la mediación y su importancia actual en la práctica pedagógica. Summa psicológica UST, 5(2), 87-96.

Mañú, J.M. \& Goyarrola, I. (2011). Docentes competentes. Por una educación de calidad. Madrid: Narcea.

Marcelo, C. \& Vaillant, D. (2009). Desarrollo profesional docente. Madrid: Narcea.

Martínez Diloné, H. A., \& González Pons, S. (2010). Acompañamiento pedagógico y profesionalización docente: sentido y perspectiva. Ciencia y sociedad, 35(3) 521-541.

Maturana, R. H. (1997). La realidad: ¿objetiva o construida? Primera reimpresión. Nueva Ciencia. España: Anthropos/ UI/ITESO.

Matos Moquete, M. (2013, 3 de enero). La hora de la formación de los maestros. Diario Libre. Sección Opinión. p.17.

Maya, A. (1993). Orientaciones básicas sobre educación a distancia y la función tutorial. San José Costa Rica: Oficina Subregional de Educación de la Unesco para Centroamérica y Panamá. Recuperado de https://bit.ly/1iiWlJ1

Mejía, R. (coord.), Castillo, G.; González, S.; Ayala, J. y Ferrand, M. L. (2005). La formación de profesores. Santo Domingo: Editora Búho.

MESCyT (2012). Programa reformulación de la formación docente. Santo Domingo: MESCyT.

MINERD (2014). Estándares profesionales y del desempeño para la certificación y desarrollo de la carrera docente. Santo Domingo: MINERD.

Osto, E. (2006). Proyecto Formativo. Guadalajara: Ediciones S.T.J.

Planela, J. (2009). Ser Educador. Entre la pedagogía y el nomadismo. Barcelona: Editorial U. O. C. Pogré, P. \& Krichesky, G. (2005). Formar docentes una alternativa multidisciplinaria. Buenos Aires: Papers Editores. 
Reimers, F. \& Tiburcio, L. (1994). Educación, ajuste y reconstrucción: opciones para el cambio. (Documento de debate de la UNESCO). París: Ediciones Unesco.

Rodríguez, W., (2013). Perfiles y perspectivas del acompañamiento: mirada crítica y claves para el desarrollo del modelo histórico-cultural. Diplomado en Acompañamiento Pedagógico, INTEC.

Santos, M. A. (2006). Enseñar o el oficio de aprender. Santa Fe: HomoSapiens.

Secretaría de Estado de Educación. (2004). Modelo de gestión de la calidad para los centros educativos. Gestión Educativa 2004-2008. Santo Domingo: SEE.

Segovia, J. (1997). Investigación educativa y formación del profesorado. Madrid: Escuela Española.

Soto, M. (2011). Acompañamiento pedagógico en Fe y Alegría. Un camino para la formación y transformación. Colección Procesos Educativo. Núm. 28. Maracaibo: Editorial Fe y Alegría.

Tamayo Guzmán, D. M. (1998). Capacidad de desempeńo del personal directivo en la gestión de centros educativos del nivel básico del Distrito Educativo 11-02 de Puerto Plata. PUCMM. Recinto Santiago de los Caballeros.

Tardif, M. (2004). Los saberes del docente y su desarrollo profesional. Madrid: Narcea.

UNESCO (Julio, 1979). En Resolución número 69 de la Conferencia Internacional de Educación de 1975. 37a. reunión llevada a cabo por la UNESCO, Ginebra, Suiza.

Valle Lima, A. D. \& García Batista, G. A. (comp.) (2007). Dirección, organización e higiene escolar. La Habana: Pueblo y Educación.

Vásquez Valerio, F. J. (2006). Modernas estrategias para la enseñanza. Ciudad de México: Euroméxico. Vigostsky, L. (1987). Historia del desarrollo de las funciones psíquicas superiores. La Habana: Editorial Científico-Técnica 